\title{
Radiative corrections to the Higgs potential in the LH model
}

\author{
Antonio Dobado $^{1}$, Lourdes Tabares-Cheluci ${ }^{1}$, Siannah Peñaranda ${ }^{2, a}$, Javier Rodriguez Laguna ${ }^{3}$ \\ ${ }^{1}$ Departamento de Física Teórica I, Universidad Complutense de Madrid, 28040 Madrid, Spain \\ ${ }^{2}$ Departamento de Física Teórica, Universidad de Zaragoza, 50009 Zaragoza, Spain \\ ${ }^{3}$ Departamento de Matemáticas, Universidad Carlos III de Madrid, 28911 Madrid, Spain
}

Received: 11 November 2009 / Revised: 24 December 2009 / Published online: 24 February 2010

(C) Springer-Verlag / Società Italiana di Fisica 2010

\begin{abstract}
In this work we compute the radiative corrections to the Higgs mass and the Higgs quartic couplings coming from the Higgs sector itself and the scalar fields $\phi$ in the Littlest Higgs (LH) model. The restrictions that the new contributions set on the parameter space of the models are also discussed. Finally, this work, together with our three previous papers, complete our program addressed to compute the relevant contributions to the Higgs low-energy effective potential in the LH model and the analysis of their phenomenological consequences.
\end{abstract}

\section{Introduction}

The discovery of a Higgs boson and the elucidation of the mechanism responsible for the electroweak symmetry breaking are some of the major goals of present and future searches in particle physics. Because of the precise data obtained for a long time to test the Standard Model (SM) of particle interactions, and the recent measurements of the $W$ and the top masses at the Fermilab Tevatron [1,2], the SM has been confirmed as the right model describing the electroweak phenomena at the current experimental energy scale. However, the origin of the electroweak symmetry breaking, for which the Higgs boson is responsible in the SM, remains elusive. The quadratically-divergent contributions to the Higgs mass and the electroweak precision observables imply different scales for physics beyond the $\mathrm{SM}$, being the first one below $1 \mathrm{TeV}$ and the second one above $10 \mathrm{TeV}$. This is the so called little hierarchy problem. As is well known the mass of the Higgs boson receives one-loop corrections that are quadratic in the loop momenta. The largest contributions come from the top quark loop, with smaller corrections coming from loops of the electroweak gauge bosons and of the Higgs boson itself. Cancellations

a e-mail: siannah@unizar.es between the top sector and other sectors must occur in order to have the Higgs mass lighter than $200 \mathrm{GeV}$ as expected from the electroweak precision test of the SM, which requires a fine-tuning of one part in 100. As this situation is quite unnatural various theories and models have been designed to solve this problem.

An interesting attempt to deal with it is the so called Littlest Higgs model (LH) [3], inspired in an old suggestion by Georgi and Pais [4, 5], which tries to solve the little hierarchy problem by adding new particles with masses $O(\mathrm{TeV})$ and symmetries which protect the Higgs mass from those dangerous quadratically-divergent contributions (see [6] and [7] for reviews). These particles include the Goldstone bosons (GB) corresponding to a global spontaneous symmetry breaking (SSB) from the $S U(5)$ to the $S O(5)$ group, a new third generation vector quark called $T$ and the gauge bosons corresponding to an additional $S U(2)$ gauge group and eventually a new hypercharge $U(1)$. In this case, and contrary to the supersymmetric theories, cancellation occurs between same-statistics particles. However, LH models typically leave uncanceled logarithmic divergences which requires additional new contributions at some higher scale to preserve a small Higgs boson mass. Many of such models with different theory spaces have been constructed [3, 8-12], and electroweak precision constraints on various little Higgs models have been investigated by performing global fits to the precision data [13-28]. The existence of the different new states in these models could give rise to a very rich phenomenology, which could be probed at the CERN Large Hadron Collider (LHC) [29, 30].

Nevertheless, it is clear that any viable model has to fulfill the basic requirement of reproducing the SM model at low energies. In particular, from the LH model it is possible in principle to compute the Higgs low-energy effective potential and then, by comparing with the SM potential, to obtain their phenomenological consequences including new restrictions on the parameter space of the LH model itself. 
For example, one can obtain the one-loop contribution to the parameters of the standard Higgs potential,

$V=-\mu^{2} H H^{\dagger}+\lambda\left(H H^{\dagger}\right)^{2} ;$

where $\mu^{2}$ and $\lambda$ denote the well known Higgs mass and Higgs self-couplings parameters. Then it is possible to set restrictions over the LH parameters space by imposing the condition $\mu^{2}=\lambda v^{2}$, where $v$ is the SM vacuum expectation value $(H=(0, v) / \sqrt{2})$. The $\mu^{2}$ sign and value are well known $[3,30]$, and effectively they are the right ones to produce the electroweak symmetry breaking, giving a Higgs mass $m_{H}^{2}=2 \mu^{2}$. However, the full expression for the radiative corrections to $\lambda$ has not been analyzed in detail so far. In principle both $\mu^{2}$ and $\lambda$ receive contributions from fermion, gauge boson and scalar loops, besides others that could come from the ultraviolet completion of the $\mathrm{LH}$ model. We have previously computed the contributions to the Higgs effective potential in the LH model coming from the fermion sector and the gauge boson sector [31, 32]. On the other hand, several relations for the threshold corrections to the $\lambda$ parameter in the presence of a $10 \mathrm{TeV}$ cut-off, depending on the UV completion of the theory, have been reported (see, for example [34]). Besides, we have computed the effective potential for the doublet Higgs and the triplet $\phi$ [33], coming from the fermionic and gauge boson oneloop contributions and from the higher order effective operators needed for the ultraviolet completion of the model.

In [31] and [32] we computed and analyzed the fermion contributions to the low-energy Higgs effective potential together with the effects of virtual heavy and electroweak gauge bosons present in the LH model. We have illustrated in these works the kind of constraints on the possible values of the LH parameters that can be set by requiring the complete LH effective potential to reproduce exactly the SM potential. The radiative corrections to $\lambda$, at the one-loop level, had not been previously computed. The computation of $\lambda$ is important for several reasons: First, it must be positive, for the low-energy effective action to make sense. In addition, from the effective potential (1.1), one gets the simple formula $m_{H}^{2}=2 \lambda v^{2}$ or, equivalently, $\mu^{2}=\lambda v^{2}$, where $v$ is set by the experiment (for instance from the muon lifetime) to be $v \simeq 245 \mathrm{GeV}$. In our phenomenological discussion in $[31,32]$ we have shown that the one-loop effective potential of the LH model cannot reproduce the SM potential with a low enough Higgs mass, $m_{H}^{2}=2 \lambda v^{2}=2 \mu^{2}$, in a range of $114 \mathrm{GeV}<m_{H}<200 \mathrm{GeV}$ in which a SM Higgs boson is expected to be found.

In order to solve this problem we computed in [33] the effective potential for the doublet Higgs and the triplet $\phi$; coming from the fermionic and gauge boson one-loop contributions and also from the higher order effective operators, as defined in [29]. The relevant terms of this effective potential can be read as

$$
\begin{aligned}
V_{\mathrm{eff}}(H, \phi) & \\
= & -\mu_{f g}^{2} H H^{\dagger}+\lambda_{f g}\left(H H^{\dagger}\right)^{2} \\
& +\lambda_{\phi^{2}} f^{2} \operatorname{tr}\left(\phi \phi^{\dagger}\right)+i \lambda_{H^{2} \phi} f\left(H \phi^{\dagger} H^{\mathrm{T}}-H^{*} \phi H^{\dagger}\right),
\end{aligned}
$$

where $\mu_{f g}^{2}>0$ and $\lambda_{f g}>0$.

With this potential we studied the regions of the LH parameter space giving rise to the SM electroweak symmetry breaking. Although radiative corrections from fermion and gauge boson loops were discussed in [31, 32], the radiative contributions to $\lambda_{\phi^{2}}$ and $\lambda_{H^{2} \phi}$ have not been computed so far. New constraints over the LH parameter space emerge once we impose the new relation between coefficients of the effective Higgs potential namely; $v^{2}=\mu_{f g}^{2} /\left(\lambda_{f g}-\lambda_{H^{2} \phi}^{2} / \lambda_{\phi^{2}}\right)$. In particular, the lowest value found for the $\mu$ parameter was $390 \mathrm{GeV}$ [33], which implied a Higgs boson mass of about $m_{H} \simeq 550 \mathrm{GeV}$, still not in the expected range for the SM Higgs boson mass.

On the other hand it is well known that the radiative corrections coming from the Higgs itself and the $\phi$ fields could also provide relevant contributions to the effective potential. Thus the main goal of the present work is to check whether these corrections could really reduce the Higgs mass to solve the above mentioned problem, making the LH model compatible with the present phenomenology. As already known, in the LH model the Higgs boson is a pseudo-Goldstone boson resulting from the spontaneous breaking of the approximate global symmetry and so is naturally light. Modifications of the standard electroweak fits, and also of the Higgs boson mass, are obtained when contributions from the extra states in the LH model are considered. One example is the case of the $\rho$ parameter, whose relation $\rho=1$ is modified at the tree level and requires an additional input parameter in the gauge-fermion sector, which can be taken to be the vacuum expectation value of the Higgs triplet, $v^{\prime}$. Many of the familiar predictions of the SM are changed by the need for an extra input parameter. However, it is still interesting to look at what parts of the $\mathrm{LH}$ parameter space yield $114 \mathrm{GeV}$ $<m_{H}<200 \mathrm{GeV}$ because this is the range in which a SM Higgs boson is expected to be found.

This work is organized as follows: In Sect. 2 we briefly explain the LH model. A summary on the SSB and the mass eigenstates is presented in Sect. 3. We set the notation in the two aforementioned sections. Section 4 is devoted to the computation of the radiative corrections contributions to the Higgs mass and quartic coupling coming from the scalar sector loops. In Sect. 5 we analyze the constraints that our computation establishes on the LH parameters and, finally, in Sect. 6 we present the conclusions. The expressions of the 
coefficients of the effective potential (1.2) coming from the radiative corrections and the effective operators are listed in the Appendix.

\section{The model}

The LH model is based on the assumption that there is a physical system with a global $S U(5)$ symmetry that is spontaneously broken to a $S O(5)$ symmetry at a high scale $\Lambda$ through a vacuum expectation value (v.e.v) of order $f$. Thus, 14 Goldstone bosons (GB) are obtained as a consequence of this breaking. In this work we will consider two different versions of the LH model. In the first one the $S U(5)$ subgroup $[S U(2) \times U(1)]^{2}$ is gauged. We refer to this version as Model I. In the second one the gauge group is $\left[S U(2)^{2} \times U(1)\right]$ (Model II) [31, 32]. In both cases some of the $\mathrm{GB}$ acquire masses through radiative corrections coming from the gauge bosons and the $t, b$ and $T$ fermions loops.

The starting Lagrangian of the LH model is given by [3, 29, 30]:

$L=L_{\Sigma}+L_{\mathrm{YK}}$,

where $L_{\Sigma}$ is the Non Linear Sigma Model (NLSM) Lagrangian:

$L_{\Sigma}=\frac{f^{2}}{8} \operatorname{tr}\left[\left(D_{\mu} \Sigma\right)\left(D^{\mu} \Sigma\right)^{\dagger}\right] ;$

and $L_{Y K}$ the Yukawa couplings for fermions and scalars:

$$
\begin{aligned}
L_{\mathrm{YK}}= & -\frac{\lambda_{1}}{2} f \bar{u}_{R} \epsilon_{m n} \epsilon_{i j k} \Sigma_{i m} \Sigma_{j n} \chi_{L k} \\
& -\lambda_{2} f \bar{U}_{R} U_{L}+\text { h.c. }
\end{aligned}
$$

In the above Lagrangians $\Sigma$ is the GB matrix given by:

$$
\Sigma=e^{2 i \Pi / f} \Sigma_{0}
$$

where $\Sigma_{0}$ can be chosen to be:

$$
\Sigma_{0}=\left(\begin{array}{lll}
0 & 0 & \mathbf{1} \\
0 & 1 & 0 \\
\mathbf{1} & 0 & 0
\end{array}\right)
$$

with 1 being the $2 \times 2$ unit matrix, and the $\Pi$ matrix can be parameterized as:

$$
\Pi=\left(\begin{array}{ccc}
0 & \frac{-i}{\sqrt{2}} H^{\dagger} & \phi^{\dagger} \\
\frac{i}{\sqrt{2}} H & 0 & \frac{-i}{\sqrt{2}} H^{*} \\
\phi & \frac{i}{\sqrt{2}} H^{\mathrm{T}} & 0
\end{array}\right),
$$

where $H=\left(H^{0}, H^{+}\right)$is the SM Higgs doublet and $\phi$ is the triplet given by:

$\phi=\left(\begin{array}{cc}\phi^{0} & \frac{1}{\sqrt{2}} \phi^{+} \\ \frac{1}{\sqrt{2}} \phi^{+} & \phi^{++}\end{array}\right)$.

The covariant derivative $D_{\mu}$ is defined as:

Model I

$$
\begin{aligned}
D_{\mu} \Sigma= & \partial_{\mu} \Sigma-i \sum_{k=1}^{2} g_{k} W_{k}^{a}\left(Q_{k}^{a} \Sigma+\Sigma Q_{k}^{a \mathrm{~T}}\right) \\
& -i \sum_{k=1}^{2} g_{k}^{\prime} B_{k}\left(Y_{k} \Sigma+\Sigma Y_{k}^{\mathrm{T}}\right),
\end{aligned}
$$

Model II

$$
\begin{aligned}
D_{\mu} \Sigma= & \partial_{\mu} \Sigma-i \sum_{k=1}^{2} g_{k} W_{k}^{a}\left(Q_{k}^{a} \Sigma+\Sigma Q_{k}^{a \mathrm{~T}}\right) \\
& -i g^{\prime} B\left(Y \Sigma+\Sigma Y^{\mathrm{T}}\right),
\end{aligned}
$$

where $g$ and $g^{\prime}$ are the gauge couplings, $W_{k}^{a}(a=1,2,3)$ and $B_{k}, B$ are the $S U(2)$ and $U(1)$ gauge fields respectively, $Q_{1 i j}^{a}=\sigma_{i j}^{a} / 2$ for $i, j=1,2$ and zero otherwise, $Q_{2 i j}^{a}=\sigma_{i-3, j-3}^{a *} / 2$ for $i, j=4,5$ and zero otherwise, $Y_{1}=$ $\operatorname{diag}(-3,-3,2,2,2) / 10, Y_{2}=\operatorname{diag}(-2,-2,-2,3,3) / 10$ and $Y=\operatorname{diag}(-1,-1,0,1,1) / 2$. The Yukawa Lagrangian in (2.3) describes the interactions between GB and fermions, more exactly, the third generations of quarks plus the extra $T$ quark appearing in the $\mathrm{LH}$ model. The indices in $L_{\mathrm{YK}}$ are defined such that $m, n=4,5, i, j=1,2,3$, and

$$
\begin{aligned}
& \bar{u}_{R}=c \bar{t}_{R}+s \bar{T}_{R}, \\
& \bar{U}_{R}=-s \bar{t}_{R}+c \bar{T}_{R},
\end{aligned}
$$

with:

$c=\cos \theta=\frac{\lambda_{2}}{\sqrt{\lambda_{1}^{2}+\lambda_{2}^{2}}}$,

$s=\sin \theta=\frac{\lambda_{1}}{\sqrt{\lambda_{1}^{2}+\lambda_{2}^{2}}}$,

and

$\chi_{L}=\left(\begin{array}{c}u \\ b \\ U\end{array}\right)_{L}=\left(\begin{array}{l}t \\ b \\ T\end{array}\right)_{L}$

In addition to the above terms it is needed to add to the $\mathrm{LH}$ Lagrangian the Yang-Mills terms corresponding to the various gauge fields, and also the gauge fixing and FaddeevPopov terms. Some of the gauge fields get massive at the 
tree level through the Higgs mechanism associated to the $S U(5) / S O(5)$ symmetry breaking. By using the Landau gauge, which is the most appropriate for the kind of computations we are presenting here (see [32] for further details), the quadratic part of the complete gauge boson Lagrangian can be written as:

$L_{\Omega}=\frac{1}{2} \Omega^{\mu}\left(\left(\square+M_{\Omega}^{2}\right) g_{\mu \nu}-\partial_{\mu} \partial_{\nu}+2 \tilde{I} g_{\mu \nu}\right) \Omega^{\nu}$,

where $\Omega$ stands for any of the gauge bosons,

Model I $\Omega^{\mu}=\left(W^{\prime \mu a}, W^{\mu a}, B^{\prime \mu}, B^{\mu}\right)$,

Model II $\Omega^{\mu}=\left(W^{\prime \mu a}, W^{\mu a}, B^{\mu}\right)$,

being the mass matrix eigenstates,

Model I $M_{\Omega}=\left(M_{W^{\prime}} 1_{3 \times 3}, 0_{3 \times 3}, M_{B^{\prime}}, 0\right)$,

Model II $\quad M_{\Omega}=\left(M_{W^{\prime}} 1_{3 \times 3}, 0_{3 \times 3}, 0\right)$,

with $M_{W^{\prime}}=f \sqrt{g_{1}^{2}+g_{2}^{2}} / 2$ and $M_{B^{\prime}}=f \sqrt{g_{1}^{\prime 2}+g_{2}^{\prime 2}} / \sqrt{20}$. The gauge boson mass eigenstates are defined such as:

$W^{a}=c_{\psi} W_{1}^{a}+s_{\psi} W_{2}^{a}$,

$W^{\prime a}=s_{\psi} W_{1}^{a}-c_{\psi} W_{2}^{a}$,

where

$s_{\psi}=\sin \psi=\frac{g_{1}}{\sqrt{g_{1}^{2}+g_{2}^{2}}}$,

$c_{\psi}=\cos \psi=\frac{g_{2}}{\sqrt{g_{1}^{2}+g_{2}^{2}}}$,

and

$B=c_{\psi}^{\prime} B_{1}+s_{\psi}^{\prime} B_{2}$,

$B^{\prime}=s_{\psi}^{\prime} B_{1}-c_{\psi}^{\prime} B_{2}$,

with

$s_{\psi}^{\prime}=\sin \psi^{\prime}=\frac{g_{1}^{\prime}}{\sqrt{g_{1}^{\prime 2}+g_{2}^{\prime 2}}}$,

$c_{\psi}^{\prime}=\cos \psi^{\prime}=\frac{g_{2}^{\prime}}{\sqrt{g_{1}^{\prime 2}+g_{2}^{\prime 2}}}$.

$\tilde{I}$ is the interaction matrix between the gauge bosons and the $H$ and $\phi$ scalars which can be found in our previous works $[32,33]$.

By adding the appropriate kinetic terms, the complete Lagrangian for the quarks becomes:

$L_{\chi}=\bar{\chi}_{R}(i \not \partial-M+\hat{I}) \chi_{L}+$ h.c., where

$$
\chi_{R}=\left(\begin{array}{l}
t \\
b \\
T
\end{array}\right)_{R},
$$

$M=\operatorname{diag}\left(0,0, m_{T}\right)$ with $m_{T}=f \sqrt{\lambda_{1}^{2}+\lambda_{2}^{2}}$ and $\hat{I}$ is the scalar-quark interaction matrix. The elements of this matrix can be found in $[31,33]$. For more details about the model, including Feynman rules and also some phenomenological results see for example [29].

\section{Effective operators}

It is well known that the effective Higgs potential receive also contributions from additional operators coming from the ultraviolet completion of the LH model. Obviously these operators must be consistent with the symmetries of the LH model $[3,29,35,40]$. At the lowest order they can be parameterized by two unknown coefficients $a$ and $a^{\prime} \sim O(1)$. The form of these effective operators is, for the fermion sector [29]:

$O_{f}=-a^{\prime} \frac{1}{4} \lambda_{1}^{2} f^{4} \epsilon^{w x} \epsilon_{y z} \epsilon^{i j k} \epsilon_{k m n} \Sigma_{i w} \Sigma_{j x} \Sigma^{* m y} \Sigma^{* n z}$,

where $i, j, k, m, n$ run over $1,2,3$ and $w, x, y, z$ run over 4, 5 and for the gauge sector we have for Model I:

$$
\begin{aligned}
O_{g b}= & \frac{1}{2} a f^{4}\left\{g_{j}^{2} \sum_{a=1}^{3} \operatorname{Tr}\left[\left(Q_{j}^{a} \Sigma\right)\left(Q_{j}^{a} \Sigma\right)^{*}\right]\right. \\
& \left.+g_{j}^{\prime 2} \operatorname{Tr}\left[\left(Y_{j} \Sigma\right)\left(Y_{j} \Sigma\right)^{*}\right]\right\},
\end{aligned}
$$

with $j=1,2$ and $Q_{j}^{a}$ and $Y_{j}$ being the generators of the $S U(2)_{j}$ and $U(1)_{j}$ groups, respectively. In the case of Model II:

$$
\begin{aligned}
O_{g b}= & \frac{1}{2} c f^{4}\left\{g_{j}^{2} \sum_{a=1}^{3} \operatorname{Tr}\left[\left(Q_{j}^{a} \Sigma\right)\left(Q_{j}^{a} \Sigma\right)^{*}\right]\right. \\
& \left.+g^{2} \operatorname{Tr}\left[(Y \Sigma)(Y \Sigma)^{*}\right]\right\},
\end{aligned}
$$

where $j=1,2$ and $Y$ is the generator of the unique $U(1)$ group.

By expanding the GB field matrix $\Sigma$ in these effective operators, we obtain their different contributions to the coefficients of the effective potential (1.2). The results are presented in the Appendix.

The complete result for the coefficients of the Higgs potential is given by the sum of the contributions coming from the effective operators, as given above, and the radiative contributions coming from all sectors of the model, as will be discussed in the following. 


\section{SSB and mass eigenstates}

In the LH model the electroweak symmetry breaking is triggered, in principle, by the Higgs potential generated by one-loop radiative corrections, including both, fermion and gauge boson loops, and the effective operators introduced in the previous section. Obviously, this potential is invariant under the electroweak gauge group $S U(2) \times U(1)$ and also should have the correct form to break this symmetry spontaneously to $U(1)_{\mathrm{em}}$. The relevant terms for this work are given in (1.2). Quartic terms involving $\phi^{4}$ and $H^{2} \phi^{2}$ are not included since they give subleading contributions to the Higgs mass. These parameters were computed in our previous works [31-33] and are given in the Appendix for completeness.

The scalar potential, as given in (1.2), reaches its minimum at: $\left\langle H H^{\dagger}\right\rangle=v^{2} / 2$ and $\left\langle\phi \phi^{\dagger}\right\rangle=v^{\prime 2}$ with:

$v^{2}=\frac{\mu_{f g}^{2}}{\lambda_{f g}-\lambda_{H^{2} \phi}^{2} / \lambda_{\phi^{2}}}$,

$v^{\prime 2}=\frac{\lambda_{H^{2} \phi}}{\sqrt{2} \lambda_{\phi^{2}}} \frac{v^{2}}{f}$.

Note that both, the doublet and triplet scalars, get a v.e.v., $v$ and $v^{\prime}$ respectively. A standard choice for the components of these fields at the vacuum is:

$$
\begin{array}{ll}
H^{+}=0, & H_{0}=\frac{v}{\sqrt{2}}, \\
\phi_{0}=-v^{\prime}, & \phi^{+}=\phi^{++}=0 .
\end{array}
$$

Then $H$ and $\phi$ can be parameterized as:

$$
\begin{aligned}
& H=\left(w^{+}, \frac{1}{\sqrt{2}}\left(v+h+i w_{0}\right)\right) \text { and } \\
& \phi=\left(\begin{array}{cc}
-v^{\prime}+\frac{1}{\sqrt{2}}(\xi+i \rho) & \frac{1}{\sqrt{2}} \phi^{+} \\
\frac{1}{\sqrt{2}} \phi^{+} & \phi^{++}
\end{array}\right) .
\end{aligned}
$$

Obviously the new fields describe fluctuations around the vacuum and the potential written in terms of them can be split in four sectors, namely, the scalar, the pseudoscalar, the charged and the doubly charged. For the first three sectors we find that the new fields are not mass eigenstates. By diagonalizing the corresponding mass matrices we obtain the mass eigenstates in each case. I.e., for the scalar sector:

$$
\begin{array}{ll}
h=c_{0} \mathcal{H}+s_{0} \Phi_{0}, & m_{\mathcal{H}}^{2} \equiv m_{f g}^{2}=2 \mu_{f g}^{2}, \\
\xi=c_{0} \Phi_{0}-s_{0} \mathcal{H}, & m_{\Phi_{0}}^{2}=M_{\phi}^{2}+2 m^{2},
\end{array}
$$

the pseudoscalar sector:

$$
\begin{aligned}
& w_{0}=c_{P} G^{0}+s_{P} \Phi^{\mathrm{P}}, \quad m_{G^{0}}^{2}=0, \\
& \rho=c_{P} \Phi^{\mathrm{P}}-s_{P} G^{0}, \quad m_{\Phi^{\mathrm{P}}}^{2}=M_{\phi}^{2}+2 m^{2},
\end{aligned}
$$

and the charged sector:

$$
\begin{array}{ll}
w^{+}=c_{+} G^{+}+s_{+} \Phi^{+}, & m_{G^{+}}^{2}=0, \\
\phi^{+}=c_{+} \Phi^{+}+s_{+} G^{+}, & m_{\Phi^{+}}^{2}=M_{\phi}^{2}+m^{2},
\end{array}
$$

with $M_{\phi}^{2}=\lambda_{\phi^{2}} f^{2}, m^{2}=v^{2} \lambda_{H^{2} \phi}^{2} / \lambda_{\phi^{2}}$. The doubly charged sector remains unchanged with a mass $M_{\phi}$.

The notation introduced for the mass eigenstates is the following: $\mathcal{H}$ and $\Phi_{0}$ are neutral scalars, $\Phi^{\mathrm{P}}$ is a neutral pseudoscalar, $\Phi^{+}$and $\Phi^{++}$are the charged and doubly charged scalars, and $G^{+}$and $G^{0}$ are the would-be Goldstone bosons corresponding to the SM $W$ and $Z$.

In terms of the mass eigenstates the leading order in the $\mathcal{O}\left(v^{2} / f^{2}\right)$ expansion of the potential is given by:

$$
\begin{aligned}
V_{\mathrm{eff}}= & \frac{1}{2} m_{f g}^{2} \mathcal{H}^{2}+\frac{1}{2} m_{\Phi_{0}}^{2} \Phi_{0}^{2}+\frac{1}{2} m_{\Phi p}^{2} \Phi^{\mathrm{P}^{2}} \\
& +m_{\Phi^{+}}^{2} \Phi^{+} \Phi^{-}+v \lambda_{f g} \mathcal{H}^{3}+v \lambda_{f g} G^{0^{2}} \mathcal{H} \\
& +2 v \lambda_{f g} G^{+} G^{-} \mathcal{H} \\
& +\frac{\lambda_{f g}}{4} \mathcal{H}^{4}+\frac{\lambda_{f g}}{2} \mathcal{H}^{2} G^{0^{2}}+\lambda_{f g} \mathcal{H}^{2} G^{+} G^{-} \\
& -\frac{\lambda_{H^{2} \phi}}{\sqrt{2}} f \mathcal{H}^{2} \Phi_{0}-\sqrt{2} \lambda_{H^{2} \phi} f \mathcal{H} G^{0} \Phi^{\mathrm{P}} \\
& -\lambda_{H^{2} \phi} f\left(\mathcal{H} G^{-} \Phi^{+}+\mathcal{H} G^{+} \Phi^{-}\right)+\cdots
\end{aligned}
$$

\section{Goldstone boson sector contributions}

The objective of this section is the computation of the radiative contributions to the Higgs mass and the Higgs quartic coupling coming from the GB sector. The relevant Lagrangian is given by:

$$
\begin{aligned}
L_{\mathrm{GB}}= & \frac{1}{2}\left(\partial_{\mu} \Pi\right)\left(\partial^{\mu} \Pi\right)+\frac{1}{f^{2}}\left(\left(\partial_{\mu} \Pi\right)\left(\partial^{\mu} \Pi\right) \Pi \Pi\right. \\
& \left.+\Pi\left(\partial_{\mu} \Pi\right) \Pi\left(\partial^{\mu} \Pi\right)\right)-V_{\mathrm{eff}} .
\end{aligned}
$$

In order to calculate the radiative contributions we write this Lagrangian in terms of the mass eigenstates and we split the Higgs field as $\mathcal{H}=\overline{\mathcal{H}}+\tilde{\mathcal{H}}$ where $\overline{\mathcal{H}}$ is the vacuum field and $\tilde{\mathcal{H}}$ describes the field fluctuations around this point. Then the first two terms of the Lagrangian above become:

$$
\begin{aligned}
L_{\text {Kin }}= & \frac{1}{2}\left(1+2 \frac{\overline{\mathcal{H}}^{2}}{f^{2}}\right)\left(\partial_{\mu} \tilde{\mathcal{H}}\right)\left(\partial^{\mu} \tilde{\mathcal{H}}\right) \\
& +\frac{1}{2}\left(1+\frac{\overline{\mathcal{H}}^{2}}{2 f^{2}}\right)\left(\partial_{\mu} \Phi_{0}\right)\left(\partial^{\mu} \Phi_{0}\right) \\
& +\frac{1}{2}\left(1+\frac{\overline{\mathcal{H}}^{2}}{2 f^{2}}\right)\left(\partial_{\mu} G^{0}\right)\left(\partial^{\mu} G^{0}\right)
\end{aligned}
$$




$$
\begin{aligned}
& +\frac{1}{2}\left(1+\frac{\overline{\mathcal{H}}^{2}}{2 f^{2}}\right)\left(\partial_{\mu} \Phi^{\mathrm{P}}\right)\left(\partial^{\mu} \Phi^{\mathrm{P}}\right) \\
& +\left(1+\frac{\overline{\mathcal{H}}^{2}}{4 f^{2}}\right)\left(\partial_{\mu} \Phi^{+}\right)\left(\partial^{\mu} \Phi^{-}\right) \\
& +\left(1+\frac{\overline{\mathcal{H}}^{2}}{2 f^{2}}\right)\left(\partial_{\mu} G^{+}\right)\left(\partial^{\mu} G^{-}\right) \\
& +\left(\partial_{\mu} \Phi^{++}\right)\left(\partial^{\mu} \Phi^{--}\right) .
\end{aligned}
$$

Obviously, all the kinetic terms in this formula, but the last one, are not properly normalized. Therefore we write the fields in terms of a new set of properly normalized fields up to order $1 / f^{2}$ as:

$$
\begin{aligned}
\Upsilon & =\left(1-\frac{\overline{\mathcal{H}}^{2}}{4 f^{2}}\right) \Upsilon^{\prime} \quad \text { with } \\
& \Upsilon^{\left({ }^{\prime}\right)}=G^{0\left(^{\prime}\right)}, G^{ \pm\left({ }^{\prime}\right)}, \Phi_{0}^{\left({ }^{\prime}\right)}, \Phi^{P\left({ }^{\prime}\right)}, \\
\tilde{\mathcal{H}} & =\left(1-\frac{\overline{\mathcal{H}}^{2}}{f^{2}}\right) \mathcal{H}^{\prime}, \\
\Phi^{ \pm} & =\left(1-\frac{\overline{\mathcal{H}}^{2}}{8 f^{2}}\right) \Phi^{ \pm \prime},
\end{aligned}
$$

so that the Lagrangian is just:

$$
\begin{aligned}
L_{\text {Kin }}= & \frac{1}{2} \partial_{\mu} \mathcal{H}^{\prime} \partial^{\mu} \mathcal{H}^{\prime} \\
& +\frac{1}{2} \partial_{\mu} \Phi_{0}^{\prime} \partial^{\mu} \Phi_{0}^{\prime}+\frac{1}{2} \partial_{\mu} G^{0 \prime} \partial^{\mu} G^{0 \prime}+\frac{1}{2} \partial_{\mu} \Phi^{\mathrm{P}^{\prime}} \partial^{\mu} \Phi^{\mathrm{P}^{\prime}} \\
& +\partial_{\mu} G^{+\prime} \partial^{\mu} G^{-\prime}+\partial_{\mu} \Phi^{+\prime} \partial^{\mu} \Phi^{-\prime} \\
& +\partial_{\mu} \Phi^{++} \partial^{\mu} \Phi^{--} .
\end{aligned}
$$

Then, the effective potential $V_{\text {eff }}$ is given by:

$$
\begin{aligned}
V_{\text {eff }}= & \frac{1}{2} m_{f g}^{2} \overline{\mathcal{H}}^{2}+\frac{\lambda_{f g}}{4} \overline{\mathcal{H}}^{4}+V_{\text {eff }}^{s s} \\
& +V_{\text {eff }}^{p s}+V_{\text {eff }}^{c s}+\cdots,
\end{aligned}
$$

where

$$
\begin{aligned}
V_{\mathrm{eff}}^{s s}= & \frac{1}{2} m_{\Phi_{0}}^{2} \Phi_{0}^{\prime 2}+\frac{1}{2} m_{f g}^{2} \mathcal{H}^{\prime 2} \\
& +\frac{3}{2} \lambda_{f g} \overline{\mathcal{H}}^{2} \mathcal{H}^{\prime 2}-\frac{\lambda_{\phi^{2}}}{4} \overline{\mathcal{H}}^{2} \Phi_{0}^{\prime 2} \\
& -\sqrt{2} \lambda_{H^{2} \phi} f \overline{\mathcal{H}} \mathcal{H}^{\prime} \Phi_{0}^{\prime}-\frac{\lambda_{H^{2} \phi}}{\sqrt{2}} f \overline{\mathcal{H}}^{2 \prime} \Phi_{0}^{\prime}, \\
V_{\mathrm{eff}}^{p s}= & \frac{1}{2} m_{\Phi^{p}}^{2} \Phi^{\mathrm{P}^{\prime 2}}+\frac{\lambda_{f g}}{2} \overline{\mathcal{H}}^{2} G^{0^{\prime 2}} \\
& -\frac{\lambda_{\phi^{2}}}{4} \overline{\mathcal{H}}^{2} \Phi^{\mathrm{P}^{\prime 2}}
\end{aligned}
$$

$$
\begin{aligned}
& -\sqrt{2} \lambda_{H^{2} \phi} f \overline{\mathcal{H}} G^{0 \prime} \Phi^{\mathrm{P}^{\prime}}, \\
V_{\mathrm{eff}}^{c s}= & m_{\Phi^{+}}^{2} \Phi^{\prime+} \Phi^{\prime-}+\lambda_{f g} \overline{\mathcal{H}}^{2} G^{\prime+} G^{\prime-} \\
& -\frac{\lambda_{\phi^{2}}}{4} \overline{\mathcal{H}}^{2} \Phi^{\prime+} \Phi^{\prime-} \\
& -\lambda_{H^{2} \phi} f \overline{\mathcal{H}}\left(G^{\prime-} \Phi^{\prime+}+G^{\prime+} \Phi^{\prime-}\right) .
\end{aligned}
$$

Observe that the third terms in (5.8), (5.9) and (5.10) describe the new interactions which come from the new normalization of the fields and the fact that the triplet boson mass is $\mathcal{O}\left(f^{2}\right)$. These interactions play a decisive role to cancel the quadratic divergences that come from the GB loops.

Finally, we can see that the split into different scalar sectors is maintained after diagonalization and normalization. This fact is important in order to simplify the computation of the radiative contributions coming from the GB. Thus we can deal with each scalar sector in an independent way being the computations in all cases similar. We illustrate this by computing the $\left(\mathcal{H}^{\prime}, \Phi_{0}^{\prime}\right)$ contribution and then we apply the same method to the other scalars. The effective action is computed by using the standard techniques [36].

\subsection{Scalar sector contribution}

The Lagrangian for the scalar sector $\left(\mathcal{H}^{\prime}, \Phi_{0}^{\prime}\right)$ is given by:

$$
\begin{aligned}
\mathcal{L}^{s s} & \left.\overline{\mathcal{H}}, \mathcal{H}^{\prime}, \Phi_{0}^{\prime}\right) \\
= & \frac{1}{2} \partial_{\mu} \mathcal{H}^{\prime} \partial^{\mu} \mathcal{H}^{\prime}+\frac{1}{2} \partial_{\mu} \Phi_{0}^{\prime} \partial^{\mu} \Phi_{0}^{\prime}-V_{\mathrm{eff}}^{s s} \\
= & \frac{1}{2} \partial_{\mu} \mathcal{H}^{\prime} \partial^{\mu} \mathcal{H}^{\prime}+\frac{1}{2} \partial_{\mu} \Phi_{0}^{\prime} \partial^{\mu} \Phi_{0}^{\prime} \\
& -\frac{1}{2} m_{f g}^{2} \mathcal{H}^{\prime 2}-\frac{1}{2} m_{\Phi_{0}}^{2} \Phi_{0}^{\prime 2} \\
& -\frac{3}{2} \lambda_{f g} \overline{\mathcal{H}}^{2} \mathcal{H}^{\prime 2} \\
& +\frac{\lambda_{\phi^{2}}}{4} \overline{\mathcal{H}}^{2} \Phi_{0}^{\prime 2}+\sqrt{2} f \lambda_{H^{2} \phi} \overline{\mathcal{H}} \mathcal{H}^{\prime} \Phi_{0}^{\prime}+\frac{\lambda_{H^{2} \phi}}{\sqrt{2}} \overline{\mathcal{H}}^{2} \Phi_{0}^{\prime} .
\end{aligned}
$$

The effective action for the $\overline{\mathcal{H}}$ is:

$e^{i S_{\mathrm{eff}}[\overline{\mathcal{H}}]}=\int\left[d \mathcal{H}^{\prime}\right]\left[d \Phi_{0}^{\prime}\right] e^{i \int d x \mathcal{L}^{s s}}$.

From the (5.11) we observe that the integration can be computed in two steps: First we concentrate on the $\Phi_{0}^{\prime}$ field and then we integrate the $\mathcal{H}^{\prime}$ field. After integrating $\Phi_{0}^{\prime}$ we get the $\mathcal{H}$ effective action:

$$
\begin{aligned}
S_{\text {eff }}^{s s}\left[\overline{\mathcal{H}}, \mathcal{H}^{\prime}\right]= & -\frac{i}{2} \operatorname{Tr} \log \left[1+G_{\Phi_{0}} \frac{\lambda_{\phi^{2}}}{2} \overline{\mathcal{H}}^{2}\right] \\
& -f^{2} \lambda_{H^{2} \phi}^{2} \int d x d y \overline{\mathcal{H}}^{2} \mathcal{H}_{x}^{\prime} G_{\Phi_{0} x y} \mathcal{H}_{y}^{\prime}
\end{aligned}
$$




$$
\begin{aligned}
& -\frac{\lambda_{H^{2} \phi}^{2}}{4} f^{2} \int d x d y G_{\Phi_{0} x y} \overline{\mathcal{H}}^{4} \delta_{y x} \\
= & -\frac{i}{2} \sum_{k=1}^{\infty} \frac{(-1)^{k+1}}{k} \operatorname{Tr}\left(G_{\Phi_{0}} \frac{\lambda_{\phi^{2}}}{2} \overline{\mathcal{H}}^{2}\right)^{k} \\
& +\tilde{I}_{2}+\tilde{I}_{4},
\end{aligned}
$$

where the $\Phi_{0}^{\prime}$ propagator is given by:

$G_{\Phi_{0}}(x, y)=\int d \tilde{k} e^{i k(x-y)} \frac{1}{k^{2}-m_{\Phi_{0}}^{2}}$,

here $\tilde{k} \equiv d^{4} k /(2 \pi)^{4}$, and

$$
\begin{aligned}
& \tilde{I}_{2}=-f^{2} \lambda_{H^{2} \phi}^{2} \int d x d y \overline{\mathcal{H}}^{2} \mathcal{H}_{x}^{\prime} G_{\Phi_{0} x y} \mathcal{H}_{y}^{\prime}, \\
& \tilde{I}_{4}=-\frac{\lambda^{2}{ }^{2} \phi}{4} f^{2} \int d x d y \overline{\mathcal{H}}^{4} \delta_{x y} G_{\Phi_{0} x y} .
\end{aligned}
$$

Observe that we have obtained three terms. The first and the third ones are $\mathcal{H}^{\prime}$ independent and they will give the $\Phi_{0}^{\prime}$ radiative contributions to the Higgs mass and the quartic coupling.

Now integrating out $\mathcal{H}^{\prime}$ we find its contribution to the $\overline{\mathcal{H}}$ effective action:

$$
\begin{aligned}
S^{s s}[\overline{\mathcal{H}}]= & -\frac{i}{2} \operatorname{Tr} \log \left[1+G_{\mathcal{H}^{\prime}}\left(-3 \lambda_{f g} \overline{\mathcal{H}}^{2}+2 \tilde{I}_{2}\right)\right] \\
= & \frac{i}{2} \sum_{k=1}^{\infty} \frac{(-1)^{k+1}}{k} \operatorname{Tr}\left(G_{\mathcal{H}}\left(3 \lambda_{f g} \overline{\mathcal{H}}^{2}-2 \tilde{I}_{2}\right)\right) \\
& +\cdots,
\end{aligned}
$$

where $G_{\mathcal{H}^{\prime}}$ is the $\mathcal{H}^{\prime}$ propagator,

$G_{\mathcal{H}^{\prime}}(x, y)=\int d \tilde{k} e^{i k(x-y)} \frac{1}{k^{2}-m_{f g}^{2}}$.

Finally, by taking into account (5.13) and (5.17), we obtain the $\overline{\mathcal{H}}$ effective action which reads:

$$
\begin{aligned}
S^{s s}[\overline{\mathcal{H}}]= & -\frac{i}{2} \sum_{k=1}^{\infty} \frac{(-1)^{k+1}}{k} \operatorname{Tr}\left(G_{\Phi_{0}} \frac{\lambda_{\phi^{2}}}{2} \overline{\mathcal{H}}^{2}\right)^{k} \\
& +\frac{i}{2} \sum_{k=1}^{\infty} \frac{(-1)^{k+1}}{k} \operatorname{Tr}\left(G_{\mathcal{H}^{\prime}}\left(3 \lambda_{f g} \overline{\mathcal{H}}^{2}+\tilde{I}_{2}\right)\right)^{k} \\
& +\tilde{I}_{4} .
\end{aligned}
$$

In order to obtain the scalar contribution to the Higgs mass we only need to consider the $k=1$ term in the expansion (5.19). The generic loop diagrams are shown in Fig. 1. Then, for $k=1$,

$$
S^{(1) s s}[\overline{\mathcal{H}}]=-\frac{i}{2} \lambda_{\phi^{2}} \int d x d y\left(G_{\Phi_{0} x y} \overline{\mathcal{H}}^{2} \delta_{y x}\right)
$$
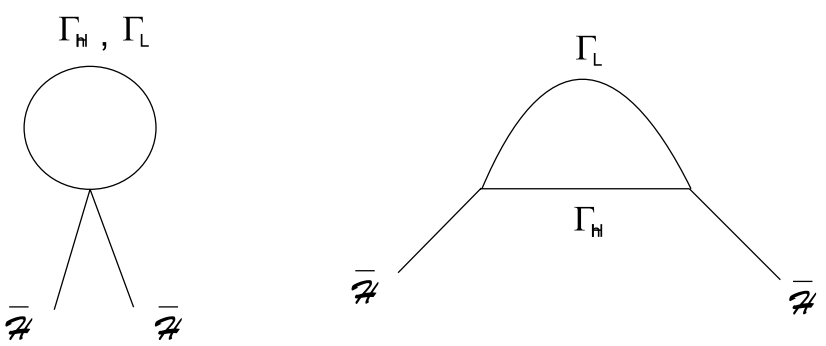

(a)

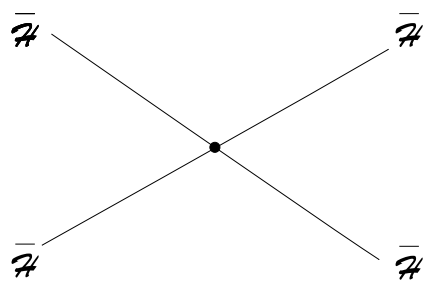

(b)

Fig. 1 a Scalar sector loops contributing to the Higgs mass. $\Gamma_{L}=\mathcal{H}^{\prime}, G^{0 \prime}$ or $G^{ \pm \prime}$ and $\Gamma_{H}=\Phi_{0}^{\prime}, \Phi^{\mathrm{P}}$ or $\Phi^{ \pm \prime}$. b Contribution to the Higgs quartic coupling from the $\Phi_{0}^{\prime}$ propagator

$$
\begin{aligned}
& +\frac{i}{2} \int d x d y G_{\mathcal{H}^{\prime} x y}\left(3 \lambda_{f g} \overline{\mathcal{H}}^{2} \delta_{y x}+\tilde{I}_{2} \delta_{y x}\right) \\
= & -\frac{i}{4} \lambda_{\phi^{2}} \int d x \overline{\mathcal{H}}^{2} I_{0}\left(m_{\Phi_{0}}^{2}\right) \\
& +\frac{3}{2} i \lambda_{f g} \int d x \overline{\mathcal{H}}^{2} I_{0}\left(m_{f g}^{2}\right) \\
& +i \lambda_{H^{2} \phi}^{2} f^{2} \int d x \overline{\mathcal{H}}^{2} I_{3}\left(m_{\Phi_{0}}^{2}, m_{f g}^{2}\right),
\end{aligned}
$$

with

$$
\begin{aligned}
I_{0}\left(M^{2}\right) \equiv \int d \tilde{k} \frac{i}{\left(k^{2}-M^{2}\right)} \\
=\frac{1}{(4 \pi)^{2}}\left[\Lambda^{2}-M^{2} \log \left(1+\frac{\Lambda^{2}}{M^{2}}\right)\right] \\
I_{3}\left(M_{a}^{2}, M_{b}^{2}\right) \equiv \int d \tilde{p} \frac{i}{\left(p^{2}-M_{a}^{2}\right)\left(p^{2}-M_{b}^{2}\right)} \\
=-\frac{1}{(4 \pi)^{2}} \frac{1}{M_{a}^{2}-M_{b}^{2}}\left[M_{a}^{2} \log \left(1+\frac{\Lambda^{2}}{M_{a}^{2}}\right)\right. \\
\left.\quad-M_{b}^{2} \log \left(1+\frac{\Lambda^{2}}{M_{b}^{2}}\right)\right] .
\end{aligned}
$$

For the quartic coupling Higgs correction coming from $\tilde{I}_{4}$ we have:

$\tilde{I}_{4}=\frac{\lambda_{H^{2} \phi}^{2}}{4 \lambda_{\phi^{2}}} \int d x \overline{\mathcal{H}}^{4}+\cdots$, 
where we have expanded the $\Phi_{0}^{\prime}$ propagator in powers of $k^{2} / m_{\Phi_{0}^{\prime}}^{2}$ and kept just the first term.

\subsection{Pseudoscalar sector and charged sector contributions}

The computation of the contributions from the pseudoscalar and charged sectors is similar to the previous ones with only one difference, i.e.: these sectors do not give a contribution to the Higgs quartic coupling. They just contribute to the Higgs mass. Then the results for the pseudoscalar sector are:

$$
\begin{aligned}
S^{(1) p s}[\overline{\mathcal{H}}]= & -\frac{i}{4} \lambda_{\phi^{2}} \int d x \overline{\mathcal{H}}^{2} I_{0}\left(m_{\Phi^{p}}^{2}\right) \\
& +\frac{1}{2} i \lambda_{f g} \int d x \overline{\mathcal{H}}^{2} I_{0}(0) \\
& +i \lambda_{H^{2} \phi}^{2} f^{2} \int d x \overline{\mathcal{H}}^{2} I_{3}\left(m_{\Phi p}^{2}, 0\right),
\end{aligned}
$$

and for the charged sector:

$$
\begin{aligned}
S^{(1) c s}[\overline{\mathcal{H}}]= & -\frac{i}{4} \lambda_{\phi^{2}} \int d x \overline{\mathcal{H}}^{2} I_{0}\left(m_{\Phi^{+}}^{2}\right) \\
& +i \lambda_{f g} \int d x \overline{\mathcal{H}}^{2} I_{0}(0) \\
& +i \lambda_{H^{2} \phi}^{2} f^{2} \int d x \overline{\mathcal{H}}^{2} I_{3}\left(m_{\Phi^{+}}^{2}, 0\right) .
\end{aligned}
$$

Notice the there is no contribution coming from the doubly charged scalar sector.

\subsection{Analytical results}

Now by adding (5.20), (5.24), (5.25) we obtain the total radiative corrections to the Higgs mass from the GB sector up to order $\mathcal{O}\left(v^{2} / f^{2}\right)$ which reads:

$$
\begin{aligned}
\Delta m_{\mathrm{GB}}^{2}= & \frac{3}{(4 \pi)^{2}}\left\{\left(-\frac{\lambda_{\phi^{2}}}{4}+\lambda_{f g}\right) \Lambda^{2}\right. \\
& +\left(\frac{\lambda_{\phi^{2}}}{4}+\frac{\lambda_{H^{2} \phi}^{2}}{\lambda_{\phi^{2}}}\right) M_{\phi}^{2} \log \left(1+\frac{\Lambda^{2}}{M_{\phi}^{2}}\right) \\
& \left.-\frac{1}{2} \lambda_{f g} m_{f g}^{2} \log \left(1+\frac{\Lambda^{2}}{m_{f g}^{2}}\right)\right\},
\end{aligned}
$$

where, in order to simplify the computations, we have considered the heavy scalar fields as degenerate since $m^{2} / M_{\phi}^{2}$ is of the order of $\mathcal{O}\left(v^{2} / f^{2}\right)$ (see (4.6)).

The coefficients of the Higgs potential $\lambda_{f g}, \lambda_{\phi^{2}}$ and $\lambda_{H^{2} \phi}$ appearing in (5.26) receive contributions from both the radiative corrections and the effective operators (see Appendix). Since the contributions to $\lambda_{f g}$ and $\lambda_{\phi^{2}}$ contain terms of the order of $\Lambda^{2}$, divergences $\mathcal{O}\left(\Lambda^{4}\right)$ and $\mathcal{O}\left(\Lambda^{2}\right)$ emerge from the first term in (5.26). However, these divergences cancel due to the relationship between $\lambda_{\phi^{2}}$ and $\lambda_{f g}$, namely:

$\lambda_{f g}^{\Lambda^{2}}=\frac{1}{4} \lambda_{\phi^{2}}^{\Lambda^{2}}$

$\lambda_{f g}^{\mathrm{EO}}=\frac{1}{4} \lambda_{\phi^{2}}^{\mathrm{EO}}$,

where the index $\Lambda^{2}$ refers to the quadratically-divergent terms and EO represents the part of these coefficients coming from the effective operators. This fact occurs in the fermionic and gauge boson sectors, where the quadratic divergences coming from light and heavy modes of the same statistics cancel [3]. Then the corrections summarized in $\Delta m_{\mathrm{GB}}^{2}(5.26)$ are at most of the order $\mathcal{O}\left(\Lambda^{2} \log \left(\Lambda^{2} / M^{2}\right)\right)$. It is important to stress that the above cancellations occur exactly only in Model I (as you can easily check from the results given in the Appendix). However, in Model II (where only the $S U(2) \times S U(2) \times U(1)$ is gauged), there are $\mathcal{O}\left(\Lambda^{2}\right)$ terms coming from the $U(1)$ sector which do not cancel. However, such terms appear always with a squared gauge coupling $g^{\prime}$ factor which is very small $\left(g^{\prime 2} / g^{2} \sim 0.3\right.$ in the $\mathrm{SM}$ ) and then their contribution is not expected to be too large.

Finally, from (5.23), the radiative correction to the quartic coupling is:

$\tilde{I}_{4}=\frac{1}{4} \Delta \lambda_{\mathrm{GB}} \int d x \overline{\mathcal{H}}^{4}$,

being

$\Delta \lambda_{\mathrm{GB}}=\frac{\lambda_{H^{2} \phi}^{2}}{\lambda_{\phi^{2}}}$.

In summary, taking into account (5.23) and (5.26), the Higgs boson potential can be written as:

$V=\frac{1}{2} m_{\overline{\mathcal{H}}}^{2} \overline{\mathcal{H}}^{2}+\frac{1}{4} \lambda \overline{\mathcal{H}} \overline{\mathcal{H}}^{4}$,

where the Higgs mass is given by,

$m_{\overline{\mathcal{H}}}^{2}=2\left(\mu_{f g}^{2}-\Delta m_{\mathrm{GB}}^{2}\right)$

and the quartic Higgs couplings is,

$\lambda \frac{2}{\mathcal{H}}=\lambda_{f g}-\frac{\lambda_{H^{2} \phi}^{2}}{\lambda_{\phi^{2}}}$.

The Higgs boson mass corrections are summarized in (5.30). The $\mu_{f g}^{2}$ parameter contains the one-loop radiative corrections coming from fermions and gauge bosons loops, but also contributions coming from the UV effective operators in the case of the Model II. $\Delta m_{\mathrm{GB}}^{2}$ summarizes the main two-loop contributions from the GB sector, 
as discussed in this section. In general, the $\mu^{2}$ parameter receives equally significant contributions from the one-loop log-divergent and two-loop quadratically-divergent parts of the Coleman-Weinberg potential [29]. Formally, only oneloop fermionic and gauge boson corrections are included in $\mu_{f g}^{2}$. However, we also discuss in this paper the role of the main two-loop GB radiative corrections, which are of the same order of magnitude as the previous one (see next section).

It is important also to note that we have obtained the GB contributions after having broken the SM symmetry through the fermion and gauge boson radiative corrections. In this fact we differ from other analysis performed in the literature (see for example [3, 35]), where these scalar contributions are computed at the tree level from the effective operators only. Moreover, in our case, the coefficients of the potential (1.2) do not depend only on the two unknown coefficients $a$ and $a^{\prime}$, but also on the scale $f$ and the cutoff $\Lambda$, thus setting more restrictions on the space parameter as we will see in the following.

\section{Numerical results and phenomenological discussion}

In this section we continue our study about the allowed region of the parameter space of the LH model started in our previous papers [31-33]. In the present one we complete this phenomenological study, taking into account also the contributions from the Goldstone boson sector to the Higgs mass and quartic coupling obtained above. The LH parameters different relationships and their relevant ranges considered are the following:

First, we impose the minimum condition for the complete effective potential (1.2):

$$
v^{2}=\frac{\mu_{f g}^{2}}{\lambda_{f g}-\lambda_{H^{2} \phi}^{2} / \lambda_{\phi^{2}}} .
$$

This condition is crucial in order to reproduce the electroweak symmetry breaking.

If we want to study the allowed region of the parameter space in these models, we should also take into account other constraints imposed by requiring the consistency of the LH models with the electroweak precision data. There exist several studies of the corrections to electroweak precision observables in the Little Higgs models, exploring whether there are regions of the parameter space in which the model is consistent with the available data [6, 7, 13-30]. In Model $I$ with a gauge group $S U(2) \times S U(2) \times U(1) \times U(1)$ we have a multiplet of heavy $S U(2)$ gauge bosons and a heavy $U(1)$ gauge boson. The last one leads to large electroweak corrections and some problems with the direct observational bounds on the $Z^{\prime}$ boson from Tevatron [13, 14]. Then, a very strong bound on the symmetry breaking scale $f, f>4 \mathrm{TeV}$ at $95 \%$ C.L, is found [13]. However, it is known that this bound is lowered to $1-2 \mathrm{TeV}$ for some region of the parameter space [14] by gauging only $S U(2) \times S U(2) \times U(1)$ (Model II). For this reason, in the following we will concentrate only on this model.

On the other hand, in order to avoid small values for the $W^{\prime}$ mass and a very strong coupling constant, we set the range of the $\psi$ mixing angle (for the $S U(2)$ group) to be $0.1<c_{\psi}<0.9$ [32]. In addition, the condition $\lambda_{T} \gtrsim 0.5$ is established from the top mass [29], setting the bounds on the couplings $\lambda_{1}, \lambda_{2} \geq m_{t} / v$ or $\lambda_{1} \lambda_{2} \geq 2\left(m_{t} / v\right)^{2}$. In order to avoid a large fine-tuning in the Higgs potential $[3,30]$ we set the condition $m_{T} \lesssim 2.5 \mathrm{TeV}$. Then, since $m_{T}$ grows linearly with $f, f$ should be less than about one $\mathrm{TeV}$ [31]. Following the restrictions on the parameters given in [32], we take $0.8 \mathrm{TeV}<f<1 \mathrm{TeV}$. Finally the usual condition $\Lambda \lesssim 4 \pi f$ is also imposed [37-39].

By using the constraints on the LH parameters given above, taking into account also that the SM Higgs mass is restricted to the range $114 \mathrm{GeV}<m_{\overline{\mathcal{H}}}<200 \mathrm{GeV}$, and by imposing the minimum condition (6.1), we analyze the available regions for the remaining $\mathrm{LH}$ parameters. To do that we include the contributions of both radiative corrections and effective operators. In fact, in order to see the role played for each of them, we consider three different cases: having just radiative corrections (RC), just effective operators (EO) and the most general case including both of them $(\mathrm{RC}+\mathrm{EO})$.

In Fig. 2 we show the allowed regions of the parameter space for the three different cases analyzed; RC (red region), $\mathrm{EO}$ (blue region) and $\mathrm{RC}+\mathrm{EO}$ (green region). In Fig. 2a we show the possible solutions to the LH model in the $\left(\Lambda, c_{\psi}, \lambda_{T}\right)$ space varying $f$ between $0.8 \mathrm{TeV}$ and $1 \mathrm{TeV}$ and by assuming that the $a$ and $a^{\prime}$ parameters are of the order of $\mathcal{O}(1)$. From these results there are two important issues to remark. First, when only radiative corrections are included we do not find any solution for the LH model if $\Lambda>6 \mathrm{TeV}$. Unfortunately, precision electroweak data rule out new strong interactions at scales below about $10 \mathrm{TeV}$. On the contrary, in the other two cases, RC + EO and EO, the possible values for the cut-off are larger. This fact implies also that the mass of the $\phi$ fields must be about $2 \mathrm{TeV}$ when the model includes only radiative corrections unlike in the other two cases where it is about $5 \mathrm{TeV}$ (see Fig. 3). In Fig. $2 \mathrm{~b}$ we show the possible values for the unknown $a$ and $a^{\prime}$ parameters. Here, the other parameters have been varied in the ranges set above. The two cases considered are $\mathrm{RC}+\mathrm{EO}$ and EO only. We find that the set of possible solutions include in both cases positive values for $a$. In the $\mathrm{RC}+\mathrm{EO}$ case we obtain large and negative values for $a^{\prime}$, whereas in the EO case $a^{\prime}$ takes small and positive values. Notice also that $a$ is always positive. This is important since 


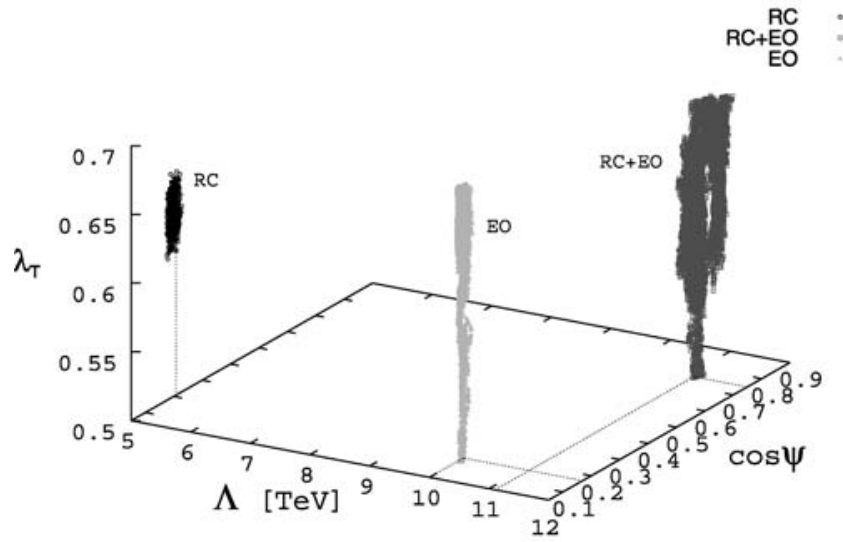

(a)

Fig. 2 a Values of $\lambda_{T}, \Lambda$ and $c_{\psi}$ which are possible solutions for the LH model. Here $f$ vary between 0.8 and $1 \mathrm{TeV}$, and $a$ and $a^{\prime}$ are $\mathcal{O}(1)$. The three separate surfaces correspond with the three different cases

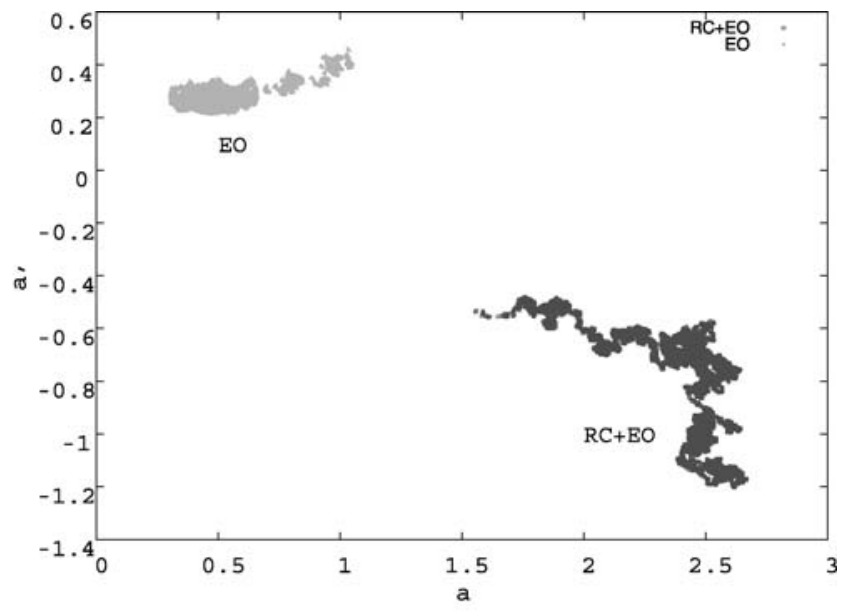

(b)

analyzed in this section. b Values of $a$ and $a^{\prime}$ which are possible solutions for the $\mathrm{LH}$ model. The $\lambda_{T}, \Lambda, c_{\psi}$ and $f$ ranges are described in the text

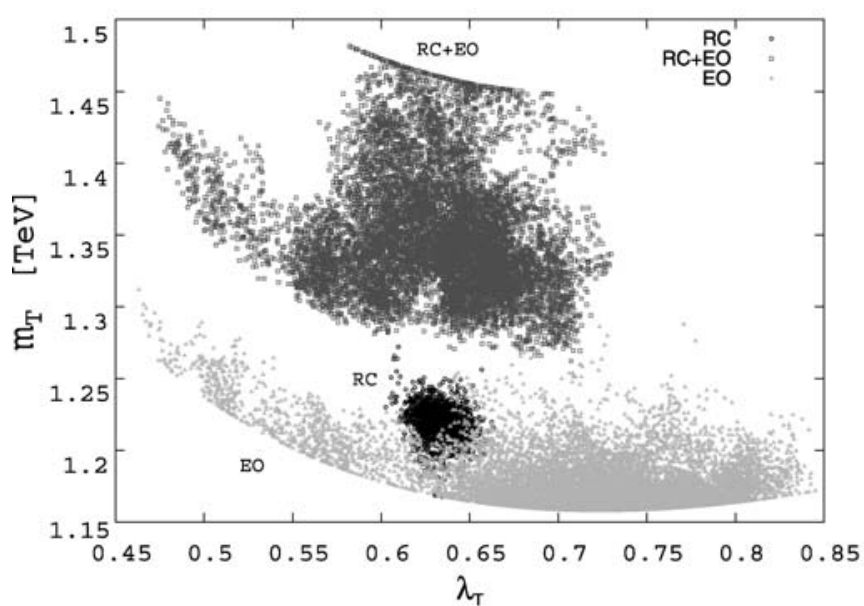

(a)

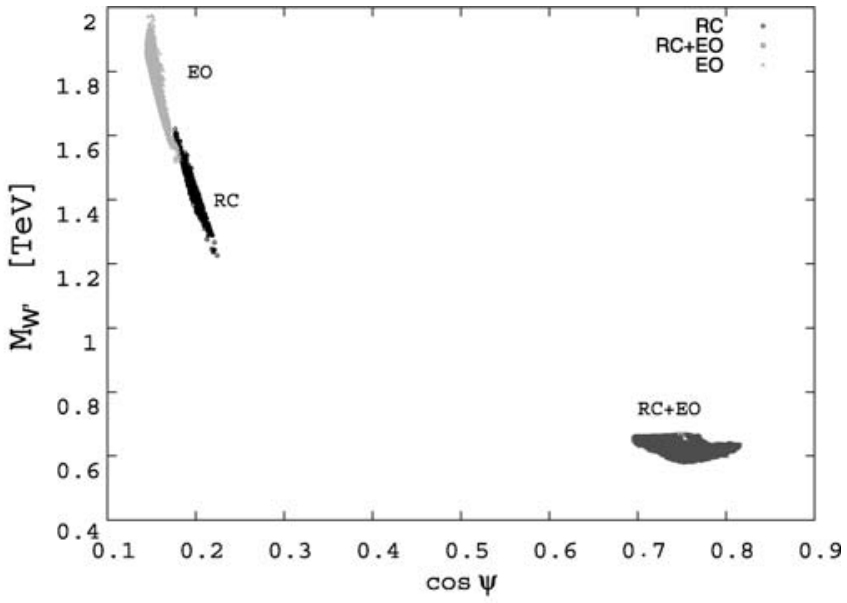

(b)

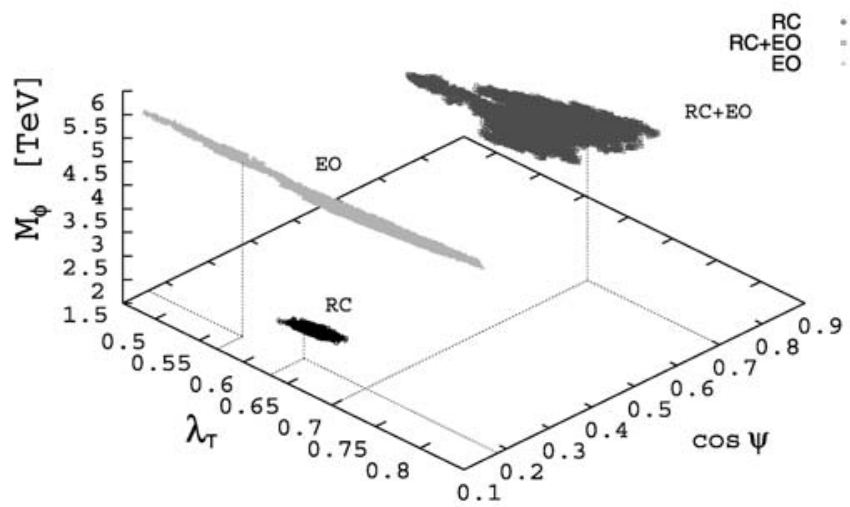

(c)

Fig. 3 a $m_{T}$ as a function of $\lambda_{T}, \mathbf{b} M_{W^{\prime}}$ as a function of $\cos \psi$ and $\mathbf{c} M_{\phi}$ as a function of $\lambda_{T}$ and $\cos \psi$, where the $\Lambda, f, a$ and $a^{\prime}$ parameters vary between ranges described in the text 
it is known that $a<0$ leads to a large v.e.v for the scalar triplet.

The reason for the differences of the parameter solutions for the three cases come from the $\Delta m_{\mathrm{GB}}^{2}$ cutoff dependence when the radiative contributions are included. For example, in the case where only the radiative corrections are taken into account, a cut-off $\Lambda$ bigger than $6 \mathrm{TeV}$ produces $\mathrm{GB}$ contributions resulting in a negative Higgs mass. However, by dropping the value of $\Lambda$ we get a LH parameter space where the condition (6.1) is satisfied and the Higgs mass is

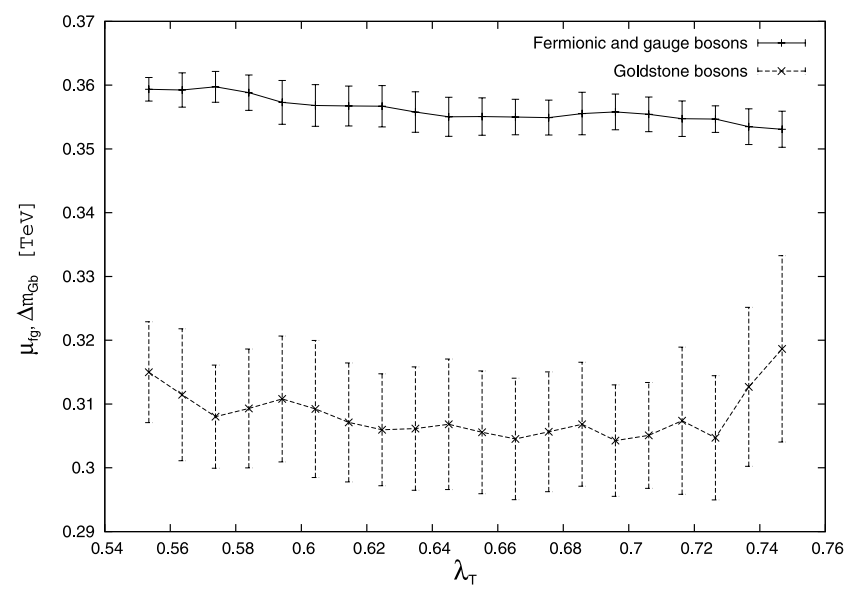

(a)

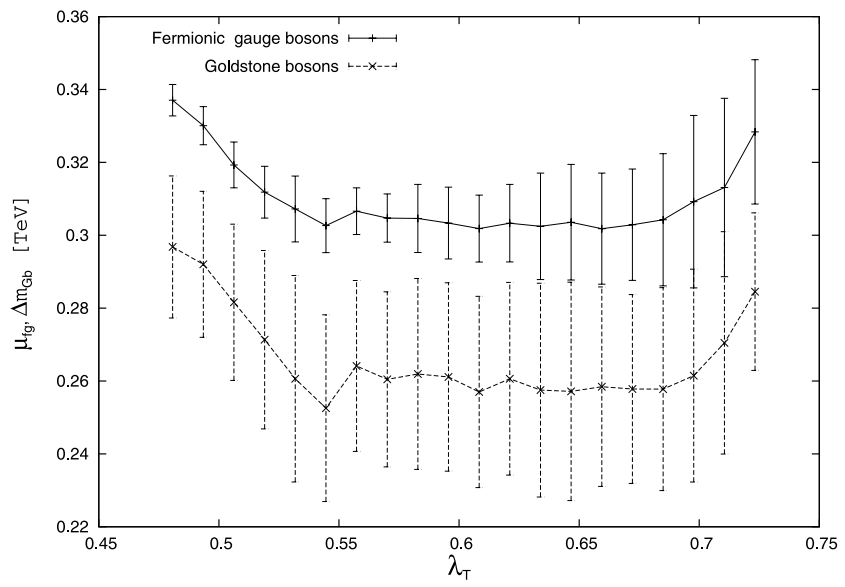

(b)

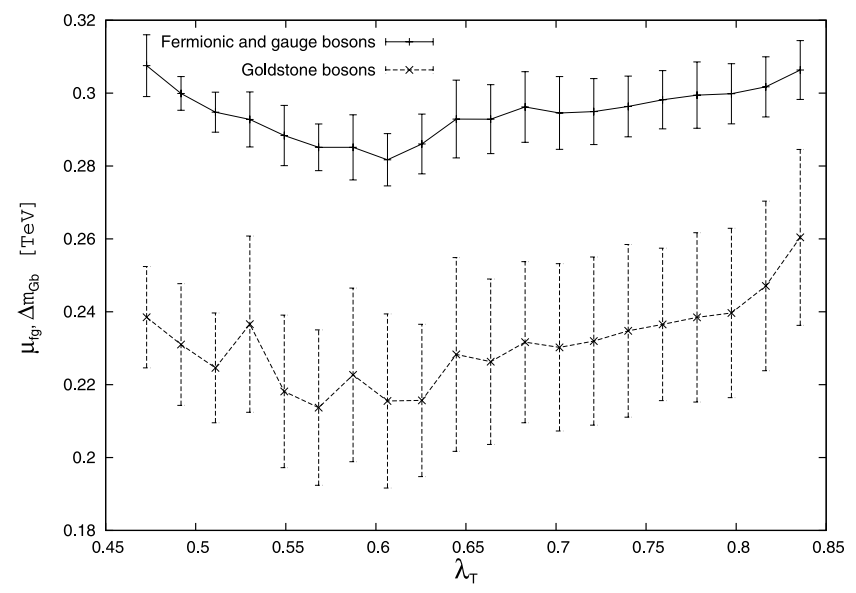

(c)

Fig. 4 These figures show the average and standard deviation of both the fermionic and gauge boson contribution $\mu_{f g}$ and the Goldstone boson contribution $\Delta m_{\mathrm{GB}}$ to the Higgs mass as a function of $\lambda_{T}$. a RC case, $\mathbf{b} \mathrm{RC}+\mathrm{EO}$ case and $\mathbf{c} \mathrm{EO}$ case

Table 1 The lowest values for the Higgs mass found for the three cases: $\mathrm{RC}, \mathrm{RC}+\mathrm{EO}$ and $\mathrm{EO}$

\begin{tabular}{llll}
\hline Parameters & RC & RC $+\mathrm{EO}$ & EO \\
\hline$m_{\overline{\mathcal{H}}}$ & $156.66 \mathrm{GeV}$ & $114.63 \mathrm{GeV}$ & $132.03 \mathrm{GeV}$ \\
$\mu_{f g}$ & $359.54 \mathrm{GeV}$ & $344.93 \mathrm{GeV}$ & $282.79 \mathrm{GeV}$ \\
$\Delta_{G b}$ & $342.04 \mathrm{GeV}$ & $335.27 \mathrm{GeV}$ & $266.94 \mathrm{GeV}$ \\
$\lambda \overline{\mathcal{H}}$ & 0.97 & 1.02 & 1.00 \\
$f$ & $0.86 \mathrm{TeV}$ & $0.91 \mathrm{TeV}$ & $0.80 \mathrm{TeV}$ \\
$\Lambda$ & $5 \mathrm{TeV}$ & $11.41 \mathrm{TeV}$ & $10.08 \mathrm{TeV}$ \\
$\lambda_{T}$ & 0.60 & 0.70 & 0.53 \\
$c_{\psi}$ & 0.18 & 0.80 & 0.15 \\
$a$ & 0 & 1.71 & 0.99 \\
$a^{\prime}$ & 0 & -0.55 & 0.4 \\
\hline
\end{tabular}


well inside the experimental constraints. In the $\mathrm{RC}+\mathrm{EO}$ case, the $a^{\prime}$ parameter can take values which help to compensate the big effect of the GB radiative contributions (see also Fig. 4) thus allowing larger cutoff values.

For completeness, Fig. 3 shows the mass values for the heavy particles in the three different cases analyzed. Each point of the figures is a possible solution of the LH model. In this way, these regions represent the possible values for the masses of the heavy particles predicted by the LH model, which are compatible with electroweak symmetry breaking and precision data. The region of possible values for the masses coming from EO contributions is larger than in the case of considering RC alone. Notice that the theoretical lower bounds in the heavy states mass, $M_{\phi} \gtrsim 1 \mathrm{TeV}$, and the condition $m_{T} \lesssim 2.5 \mathrm{TeV}$ are fulfilled.

To complete our study, we compare the contributions to the Higgs mass coming from the different sectors i.e. fermionic and gauge bosons $\left(\mu_{f g}\right)$ and on the other hand the GB contribution $\left(\Delta m_{\mathrm{GB}}\right)$, as a function of $\lambda_{T}$. We show the average and standard deviation for each contribution (Fig. 4). In all physical cases it can be seen that $\mu_{f g}>\Delta m_{\mathrm{GB}}$, thus yielding a real value for the Higgs mass (5.30). It is clear that the cancellation between fermionic and gauge bosons one-loop corrections and the two-loop GB contributions is crucial for reducing the Higgs boson mass up to the range of values expected in the SM. Notice that these two contributions are of the same order of magnitude, as expected [29]. It is also remarkable the higher variability of $\Delta m_{\mathrm{GB}}$ compared with $\mu_{f g}$. The reason is that both the parameters appearing in the radiative corrections, i.e. $f, \Lambda, \lambda_{T}, \cos \phi$, and the two EO parameters $a$ and $a^{\prime}$, play an important role in the final results of $\Delta m_{\mathrm{GB}}$ (see the discussion above).

Finally, as an example, we give in the Table 1 the lowest Higgs mass values found for the three cases considered in this work.

\section{Conclusion}

In this work we have completed our program of computing the relevant contributions to the Higgs low-energy effective potential in the context of the Littlest Higgs models based on the $S U(5) / S O(5)$ coset. To the radiative corrections coming from the fermions and the gauge bosons considered so far, we have added here the effect of the scalar loops and also the effective operators emerging from the ultraviolet completion of the model.

In particular we have computed in detail the main contributions to the Higgs mass and its quartic coupling. From our previous works, in which only fermionic and gauge boson radiative corrections were included, it was clear that the effect of the scalar sector could be decisive in order to have the appropriate cancellations between the different sectors of the model to give a Higgs mass within the present experimental limits. We have performed our analytical computations for two different versions of the model called Model I and Model II having as gauge groups $[S U(2) \times U(1)]^{2}$ and $S U(2)^{2} \times U(1)$ respectively.

In order to complete our analysis, we have concentrated on studying those regions of the parameter space where the model could give rise to an acceptable phenomenology. In particular we have done a detailed numerical search for Model II since Model I seems to be incompatible with the present experimental data [13,14]. We have analyzed three cases: (1) radiative corrections only (RC), (2) radiative corrections and effective operators ( $\mathrm{RC}+\mathrm{EO})$ and (3) effective operator only (EO). From this analysis we get that this model is compatible with the expected Higgs mass provided that the contribution of the effective operators is included. We also conclude that the Goldstone boson contributions are fundamental to obtain a low enough Higgs particle mass. For example a Higgs mass $m_{H} \simeq 115 \mathrm{GeV}$ can be obtained when radiative and effective operator contributions are both taken into account.

Summarizing, we have arrived to the conclusion that the $S U(5) / S(5)$ Littlest Higgs model with gauge group $[S U(2) \times U(1)]^{2}$ is phenomenologically viable through some tuning in the parameter space, assuming a careful inclusion of fermions, gauge bosons, scalar loops and effective operators.

In any case it will be the LHC, whose main goal is to disentangle the mechanism of the electroweak symmetry breaking, which will decide if Littlest Higgs models are appropriate for describing mechanism or not.

Acknowledgements This work is supported by DGICYT (Spain) under project number FPA2008-00592 and by the Universidad Complutense/CAM: UCM-BSCH GR58/08 910309. The work of S.P. is supported by a Ramón y Cajal contract from MEC (Spain) and partially by CICYT (grant FPA2006-2315) and DGIID-DGA (grant 2008E24/2). The work of J.R.L. is supported by project number FIS200604885. We would like to thank J.R. Espinosa for useful discussions.

\section{Appendix}

A.1 Coefficients coming from loops computation

\section{Model I}

$$
\begin{aligned}
\mu_{f g}^{2}= & \mu_{f}^{2}+\mu_{g}^{2} \\
= & 3 \frac{m_{T}^{2} \lambda_{t}^{2}}{4 \pi^{2}} \log \left(1+\frac{\Lambda^{2}}{m_{T}^{2}}\right) \\
& -\frac{3}{64 \pi^{2}}\left[3 g^{2} M_{W^{\prime}}^{2} \log \left(1+\frac{\Lambda^{2}}{M_{W^{\prime}}^{2}}\right)\right. \\
& \left.+g^{\prime 2} M_{B^{\prime}}^{2} \log \left(1+\frac{\Lambda^{2}}{M_{B^{\prime}}^{2}}\right)\right],
\end{aligned}
$$




$$
\begin{aligned}
& \lambda_{f g}=\frac{3}{(4 \pi)^{2}}\left[2\left(\lambda_{t}^{2}+\lambda_{T}^{2}\right) \frac{\Lambda^{2}}{f^{2}}\right. \\
& +\frac{3}{4(4 \pi f)^{2}}\left[\left(\frac{g^{2}}{c_{\psi}^{2} s_{\psi}^{2}}+\frac{g^{\prime 2}}{c_{\psi}^{\prime 2} s_{\psi}^{\prime 2}}\right) \Lambda^{2}\right. \\
& -\log \left(1+\frac{\Lambda^{2}}{m_{T}^{2}}\right)\left(-\frac{2 m_{T}^{2}}{f^{2}}\left(\frac{5}{3} \lambda_{t}^{2}+\lambda_{T}^{2}\right)\right. \\
& \left.+4 \lambda_{t}^{4}+4\left(\lambda_{T}^{2}+\lambda_{t}^{2}\right)^{2}\right) \\
& -4 \lambda_{T}^{2} \frac{1}{1+\frac{m_{T}^{2}}{\Lambda^{2}}}\left(\frac{m_{T}^{2}}{f^{2}}-2 \lambda_{t}^{2}-\lambda_{T}^{2}\right) \\
& \left.-4 \lambda_{t}^{4} \log \left(\frac{\Lambda^{2}}{m^{2}}\right)\right] \\
& -\frac{3}{(16 \pi f)^{2}}\left[-\left(\frac{g^{2}}{c_{\psi}^{2} s_{\psi}^{2}}+\frac{g^{\prime 2}}{c_{\psi}^{\prime 2} s_{\psi}^{\prime 2}}\right) \Lambda^{2}\right. \\
& +g^{2} M_{W^{\prime}}^{2} \log \left(1+\frac{\Lambda^{2}}{M_{W^{\prime}}^{2}}\right)\left(4+\frac{1}{c_{\psi}^{2} s_{\psi}^{2}}\right. \\
& \left.+2 g^{\prime 2} \frac{\left(c_{\psi}^{2} s_{\psi}^{\prime 2}+s_{\psi}^{2} c_{\psi}^{\prime 2}\right)^{2}}{c_{\psi}^{2} s_{\psi}^{2} c_{\psi}^{\prime 2} s_{\psi}^{\prime 2}} \frac{f^{2}}{M_{W^{\prime}}^{2}-M_{B^{\prime}}^{2}}\right) \\
& +g^{\prime 2} M_{B^{\prime}}^{2} \log \left(1+\frac{\Lambda^{2}}{M_{B^{\prime}}^{2}}\right)\left(\frac{4}{3}+\frac{1}{c_{\psi}^{\prime 2} s_{\psi}^{\prime 2}}\right. \\
& \left.+2 g^{2} \frac{\left(c_{\psi}^{2} s_{\psi}^{\prime 2}+s_{\psi}^{2} c_{\psi}^{\prime 2}\right)^{2}}{c_{\psi}^{2} s_{\psi}^{2} c_{\psi}^{\prime 2} s_{\psi}^{\prime 2}} \frac{f^{2}}{M_{B^{\prime}}^{2}-M_{W^{\prime}}^{2}}\right) \\
& +f^{2} \log \left(1+\frac{\Lambda^{2}}{M_{W^{\prime}}^{2}}\right)\left(3 g^{4}\right. \\
& \left.+2\left(3 g^{2}+g^{\prime 2}\right) g^{2} \frac{\left(s_{\psi}^{2}-c_{\psi}^{2}\right)^{2}}{c_{\psi}^{2} s_{\psi}^{2}}\right) \\
& +f^{2} \log \left(1+\frac{\Lambda^{2}}{M_{B^{\prime}}^{2}}\right)\left(g^{\prime 4}\right. \\
& \left.+2\left(g^{2}+g^{\prime 2}\right) g^{\prime 2} \frac{\left(s_{\psi}^{\prime 2}-c_{\psi}^{\prime 2}\right)^{2}}{c_{\psi}^{\prime 2} s_{\psi}^{\prime 2}}\right) \\
& +f^{2} \log \left(\frac{\Lambda^{2}}{m^{2}}\right)\left(3 g^{4}+g^{\prime 4}+8 g^{2} g^{\prime 2}\right) \\
& \left.-3 f^{2} \frac{g^{4}}{1-\frac{M_{W^{\prime}}^{2}}{\Lambda^{2}}}-f^{2} \frac{g^{\prime 4}}{1-\frac{M_{B^{\prime}}^{2}}{\Lambda^{2}}}\right] \text {, } \\
& \lambda_{\phi^{2} f}=\frac{24}{(4 \pi f)^{2}}\left(\lambda_{t}^{2}+\lambda_{T}^{2}\right)\left(\Lambda^{2}\right. \\
& \left.-m_{T}^{2} \log \left(\frac{\Lambda^{2}}{m_{T}^{2}}+1\right)\right) \\
& -g^{2} M_{W^{\prime}}^{2} \log \left(\frac{\Lambda^{2}}{M_{W^{\prime}}^{2}}+1\right)\left(\frac{\left(s_{\psi}^{2}-c_{\psi}^{2}\right)^{2}}{c_{\psi}^{2} s_{\psi}^{2}}-4\right) \\
& \left.-g^{\prime 2} M_{B^{\prime}}^{2} \log \left(\frac{\Lambda^{2}}{M_{B^{\prime}}^{2}}+1\right) \frac{\left(s_{\psi^{\prime}}^{2}-c_{\psi^{\prime}}^{2}\right)^{2}}{c_{\psi^{\prime}}^{2} s_{\psi^{\prime}}^{2}}\right], \\
& \lambda_{H^{2} \phi}=-\frac{12}{(4 \pi f)^{2}}\left[\left(\lambda_{t}^{2}+\lambda_{T}^{2}\right) \Lambda^{2}\right. \\
& \left.-\lambda_{T}^{2} m_{T}^{2} \log \left(\frac{\Lambda^{2}}{m_{T}^{2}}+1\right)\right] \\
& -\frac{3}{8(4 \pi f)^{2}}\left[g ^ { 2 } \frac { c _ { \psi } ^ { 2 } - s _ { \psi } ^ { 2 } } { c _ { \psi } ^ { 2 } s _ { \psi } ^ { 2 } } \left(\Lambda^{2}\right.\right. \\
& \left.-M_{W^{\prime}}^{2} \log \left(\frac{\Lambda^{2}}{M_{W^{\prime}}^{2}}+1\right)\right) \\
& +g^{\prime 2} \frac{c_{\psi^{\prime}}^{2}-s_{\psi^{\prime}}^{2}}{c_{\psi^{\prime}}^{2} s_{\psi^{\prime}}^{2}}\left(\Lambda^{2}\right. \\
& \left.\left.-M_{B^{\prime}}^{2} \log \left(\frac{\Lambda^{2}}{M_{B^{\prime}}^{2}}+1\right)\right)\right] \text {. } \\
& \mu_{f g}^{2}=\mu_{f}^{2}+\mu_{g}^{2} \\
& =3 \frac{m_{T}^{2} \lambda_{t}^{2}}{4 \pi^{2}} \log \left(1+\frac{\Lambda^{2}}{m_{T}^{2}}\right)-\frac{3}{64 \pi^{2}} \\
& \times\left(3 g^{2} M_{W^{\prime}}^{2} \log \left(1+\frac{\Lambda^{2}}{M_{W^{\prime}}^{2}}\right)+g^{2} \Lambda^{2}\right), \\
& \lambda_{f g}=\frac{3}{(4 \pi)^{2}}\left[2\left(\lambda_{t}^{2}+\lambda_{T}^{2}\right) \frac{\Lambda^{2}}{f^{2}}\right. \\
& -\log \left(1+\frac{\Lambda^{2}}{m_{T}^{2}}\right)\left(-\frac{2 m_{T}^{2}}{f^{2}}\left(\frac{5}{3} \lambda_{t}^{2}+\lambda_{T}^{2}\right)\right. \\
& \left.+4 \lambda_{t}^{4}+4\left(\lambda_{T}^{2}+\lambda_{t}^{2}\right)^{2}\right) \\
& -4 \lambda_{T}^{2} \frac{1}{1+\frac{m_{T}^{2}}{\Lambda^{2}}}\left(\frac{m_{T}^{2}}{f^{2}}-2 \lambda_{t}^{2}-\lambda_{T}^{2}\right) \\
& \left.-4 \lambda_{t}^{4} \log \left(\frac{\Lambda^{2}}{m^{2}}\right)\right] \\
& -\frac{3}{(16 \pi f)^{2}}\left[-\frac{g^{2}}{c_{\psi}^{2} s_{\psi}^{2}} \Lambda^{2}+\frac{4}{3} g^{\prime 2} \Lambda^{2}\right.
\end{aligned}
$$




$$
\begin{aligned}
& +g^{2} M_{W^{\prime}}^{2} \log \left(\frac{\Lambda^{2}}{M_{W^{\prime}}^{2}}+1\right)\left(4+\frac{1}{c_{\psi}^{2} s_{\psi}^{2}}\right) \\
& +f^{2} \log \left(1+\frac{\Lambda^{2}}{M_{W^{\prime}}^{2}}\right)\left(3 g^{4}\right. \\
& \left.+2\left(3 g^{2}+g^{\prime 2}\right) g^{2} \frac{\left(s_{\psi}^{2}-c_{\psi}^{2}\right)^{2}}{s_{\psi}^{2} c_{\psi}^{2}}\right) \\
& +f^{2} \log \left(\frac{\Lambda^{2}}{m^{2}}\right)\left(3 g^{4}+g^{\prime 4}\right. \\
& \left.\left.+8 g^{2} g^{\prime 2}\right)-3 f^{2} \frac{g^{4}}{1-\frac{M_{W^{\prime}}^{2}}{\Lambda^{2}}}\right] \\
& \lambda_{\phi^{2}}=\frac{24}{(4 \pi f)^{2}}\left(\lambda_{t}^{2}+\lambda_{T}^{2}\right) \\
& \times\left(\Lambda^{2}-m_{T}^{2} \log \left(\frac{\Lambda^{2}}{m_{T}^{2}}+1\right)\right) \\
& +\frac{3}{64 \pi^{2} f^{2}}\left[\frac{g^{2}}{c_{\psi}^{2} s_{\psi}^{2}} \Lambda^{2}-g^{2} M_{W^{\prime}}^{2} \log \left(\frac{\Lambda^{2}}{M_{W^{\prime}}^{2}}+1\right)\right. \\
& \left.\times\left(\frac{\left(s_{\psi}^{2}-c_{\phi}^{2}\right)^{2}}{c_{\psi}^{2} s_{\psi}^{2}}-4\right)\right] \\
& +\frac{3 g^{\prime 2}}{(4 \pi f)^{2}} \Lambda^{2} \text {, } \\
& \lambda_{H^{2} \phi}=-\frac{12}{(4 \pi f)^{2}}\left[\left(\lambda_{t}^{2}+\lambda_{T}^{2}\right) \Lambda^{2}\right. \\
& \left.-\lambda_{T}^{2} m_{T}^{2} \log \left(\frac{\Lambda^{2}}{m_{T}^{2}}+1\right)\right] \\
& -\frac{3 g^{2}}{8(4 \pi f)^{2}} \frac{c_{\psi}^{2}-s_{\psi}^{2}}{c_{\psi}^{2} s_{\psi}^{2}}\left(\Lambda^{2}\right. \\
& \left.-M_{W^{\prime}}^{2} \log \left(\frac{\Lambda^{2}}{M_{W^{\prime}}^{2}}+1\right)\right) \text {. }
\end{aligned}
$$

A.2 Coefficients coming from effective operators

\section{Model I}

$$
\begin{aligned}
& \lambda_{f g}^{\mathrm{EO}}=\frac{a}{8}\left(\frac{g^{2}}{s_{\psi}^{2} c_{\psi}^{2}}+\frac{g^{\prime 2}}{s_{\psi}^{\prime 2} c_{\psi}^{\prime 2}}\right)+a^{\prime}\left(\lambda_{t}^{2}+\lambda_{T}^{2}\right), \\
& \lambda_{\phi^{2}}^{\mathrm{EO}}=\frac{a}{2}\left(\frac{g^{2}}{s_{\psi}^{2} c_{\psi}^{2}}+\frac{g^{\prime 2}}{s_{\psi}^{\prime 2} c_{\psi}^{\prime 2}}\right)+4 a^{\prime}\left(\lambda_{t}^{2}+\lambda_{T}^{2}\right), \\
& \lambda_{H^{2} \phi}^{\mathrm{EO}}=-\frac{a}{4}\left(g^{2} \frac{c_{\psi}^{2}-s_{\psi}^{2}}{s_{\psi}^{2} c_{\psi}^{2}}+g^{\prime 2} \frac{c_{\psi}^{\prime 2}-s_{\psi}^{\prime 2}}{s_{\psi}^{\prime 2} c_{\psi}^{\prime 2}}\right)
\end{aligned}
$$

$$
-2 a^{\prime}\left(\lambda_{t}^{2}+\lambda_{T}^{2}\right)
$$

\section{Model II}

$$
\begin{aligned}
& \lambda_{f g}^{\mathrm{EO}}=\frac{a}{8}\left(\frac{g^{2}}{s_{\psi}^{2} c_{\psi}^{2}}\right)-\frac{a}{6} g^{\prime 2}+a^{\prime}\left(\lambda_{t}^{2}+\lambda_{T}^{2}\right), \\
& \lambda_{\phi^{2}}^{\mathrm{EO}}=\frac{a}{2}\left(\frac{g^{2}}{s_{\psi}^{2} c_{\psi}^{2}}\right)+4 a g^{\prime 2}+4 a^{\prime}\left(\lambda_{t}^{2}+\lambda_{T}^{2}\right), \\
& \lambda_{H^{2} \phi}^{\mathrm{EO}}=-\frac{a}{4} g^{2} \frac{c_{\psi}^{2}-s_{\psi}^{2}}{s_{\psi}^{2} c_{\psi}^{2}}-2 a^{\prime}\left(\lambda_{t}^{2}+\lambda_{T}^{2}\right), \\
& \mu^{2 \mathrm{EO}}=\frac{1}{2} a f^{2} g^{\prime 2} .
\end{aligned}
$$

\section{References}

1. T. Aaltonen et al. (CDF Collaboration), Phys. Rev. Lett. 99, 151801 (2007). arXiv:0707.0085 [hep-ex]

2. The Tevatron Electroweak Working Group (Tevatron Electroweak Working Group and CDF Collaboration and D0 Collab), arXiv:0803.1683 [hep-ex]

3. N. Arkani-Hamed, A.G. Cohen, E. Katz, A.E. Nelson, J. High Energy Phys. 0207, 034 (2002). hep-ph/0206021

4. H. Georgi, A. Pais, Phys. Rev. D 10, 539 (1974)

5. H. Georgi, A. Pais, Phys. Rev. D 12, 508 (1975)

6. M. Schmaltz, D. Tucker-Smith, Annu. Rev. Nucl. Part. Sci. 55, 229 (2005). hep-ph/0502182

7. M. Perelstein, Prog. Part. Nucl. Phys. 58, 247 (2007). hep-ph/0512128

8. M. Schmaltz, J. High Energy Phys. 0408, 056 (2004). hep-ph/ 0407143

9. H.C. Cheng, I. Low, J. High Energy Phys. 0408, 061 (2004). hep-ph/0405243

10. D.E. Kaplan, M. Schmaltz, J. High Energy Phys. 0310, 039 (2003). hep-ph/0302049

11. H.C. Cheng, I. Low, J. High Energy Phys. 0309, 051 (2003). hep-ph/0308199

12. I. Low, W. Skiba, D. Tucker-Smith, Phys. Rev. D 66, 072001 (2002). hep-ph/0207243

13. C. Csaki, J. Hubisz, G.D. Kribs, P. Meade, J. Terning, Phys. Rev. D 67, 115002 (2003). hep-ph/0211124

14. C. Csaki, J. Hubisz, G.D. Kribs, P. Meade, J. Terning, Phys. Rev. D 68, 035009 (2003). hep-ph/0303236

15. Z. Han, W. Skiba, Phys. Rev. D 72, 035005 (2005). hep-ph/ 0506206

16. H.E. Logan, Phys. Rev. D 70, 115003 (2004). hep-ph/0405072

17. T. Han, H.E. Logan, B. McElrath, L.T. Wang, Phys. Lett. B 563, 191 (2003) [Erratum-ibid. B 603, 257 (2004)]. hep-ph/0302188

18. J.L. Hewett, F.J. Petriello, T.G. Rizzo, J. High Energy Phys. 0310, 062 (2003). hep-ph/0211218

19. T. Gregoire, D.R. Smith, J.G. Wacker, Phys. Rev. D 69, 115008 (2004). hep-ph/0305275

20. M.C. Chen, S. Dawson, Phys. Rev. D 70, 015003 (2004). hep-ph/0311032

21. W. Kilian, J. Reuter, Phys. Rev. D 70, 015004 (2004). hep-ph/0311095

22. G. Marandella, C. Schappacher, A. Strumia, Phys. Rev. D 72, 035014 (2005). hep-ph/0502096 
23. S.K. Kang, C.S. Kim, J. Park, Phys. Lett. B 666, 38 (2008). arXiv:0805.0179 [hep-ph]

24. M.C. Chen, Mod. Phys. Lett. A 21, 621 (2006). hep-ph/0601126

25. S.R. Choudhury, A.S. Cornell, N. Gaur, A. Goyal, hep-ph/ 0604162

26. J.A. Conley, J. Hewett, M.P. Le, Phys. Rev. D 72, 115014 (2005). hep-ph/0507198

27. C.O. Dib, R. Rosenfeld, A. Zerwekh, AIP Conf. Proc. 815, 296 (2006). hep-ph/0509013

28. Z. Berezhiani, P.H. Chankowski, A. Falkowski, S. Pokorski, Phys. Rev. Lett. 96, 031801 (2006). hep-ph/0509311

29. T. Han, H.E. Logan, B. McElrath, L.-T. Wang, Phys. Rev. D 67, 095005 (2003). hep-ph/0301040

30. M. Perelstein, M.E. Peskin, A. Pierce, Phys. Rev. D 69, 075002 (2004). hep-ph/0310039

31. A. Dobado, L. Tabares, S. Peñaranda, Eur. Phys. J. C 50, 647 (2007). hep-ph/0606031
32. A. Dobado, L. Tabares, S. Peñaranda, Phys. Rev. D 75, 083527 (2007). hep-ph/0612131

33. A. Dobado, L. Tabares, S. Peñaranda, Eur. Phys. J. C 58, 471 (2008). arXiv:0711.4471 [hep-ph]

34. F. Bazzocchi, M. Fabbrichesi, M. Piai, Phys. Rev. D 72, 095019 (2005). hep-ph/0506175

35. J.A. Casas, J.R. Espinosa, I. Hidalgo, J. High Energy Phys. 0503, 038 (2005)

36. A. Dobado, A. Gómez-Nicola, A.L. Maroto, J.R. Peláez, Effective Lagrangians for the Standard Model (Springer, Heidelberg, 1997)

37. A. Manohar, H. Georgi, Nucl. Phys. B 234, 189 (1984)

38. M.A. Luty, Phys. Rev. D 57, 1531 (1998). hep-ph/9706235

39. A.G. Cohen, D.B. Kaplan, A.E. Nelson, Phys. Lett. B 412, 301 (1997)

40. J.R. Espinosa, J.M. No, J. High Energy Phys. 0701, 006 (2007). hep-ph/0610255 